\title{
Cardiac Lead Procedure
}

National Cancer Institute

\section{Source}

National Cancer Institute. Cardiac Lead Procedure. NCI Thesaurus. Code C99934.

A procedure involving one or more wires used for pacemaker or implantable defibrillator devices. (ACC) 